\author{
Vasil MATEICHYK ${ }^{1}$ \\ Viktoria HRUT'BA ${ }^{2}$ \\ Vadym ZIUZIUN ${ }^{3}$
}

\title{
ОЦЕНКА ЭФФЕКТИВНОСТИ ПРОГРАММЫ ЭКОЛОГИЧЕСКОЙ ЛОГИСТИКИ ПРЕДПРИЯТИЯ
}

\begin{abstract}
В работе рассмотрены общие подходы и методы оценки качества выполнения программ. Проанализированы особенности внедрения программы экологической логистики транспортных предприятий. Разработаны основные показатели оценки эффективности реализации программы.
\end{abstract}

\section{1. ВВЕДЕНИЕ}

Программа экологической логистики предприятия предусматривает внедрение логистической системы обращения с отходами за счет решения задачи многокритериальной оптимизации при наличии нескольких целевых функций минимизация негативного влияния отходов на окружающую среду с максимизацией эффективности логистических процессов обращения с отходами самого предприятия [1].

Определение параметров оптимизации логистического процесса позволит обеспечить сбор, своевременное обезвреживание и удаление отходов, соблюдение правил экологической безопасности при обращении с ними; сведение к минимуму образование отходов и уменьшение уровня их опасности, обеспечение комплексного использования материально-сырьевых ресурсов; будет способствовать максимизации утилизации отходов путем прямого или повторного использования ресурсоценных отходов, обеспечить безопасное удаление отходов, не подлежащих утилизации, путем внедрения соответствующих технологий, экологически безопасных методов и средств обращения с отходами.

Важным обязательным элементом реализации программы является установление показателей эффективности программ - количественных и качественных параметров, которые будут использоваться для оценки результативности программы и определения степени достижения целей. Показатели выполнения программы позволяет прогнозировать показатели качества или конечный результат, который общество получает в результате выполнения программы. Поэтому система оценки показателей является основой для планирования будущих действий. Она дает возможность определить причинно-следственные связи между оценкой, направлением деятельности и выделенным финансированием.

\footnotetext{
${ }^{1}$ Dr hab Vasyl Mateichyk, profesor, Widzial Zarzadzania i Marketingu, Politechnika Rzeszowska ${ }^{2}$ Dr Viktoria Hrut'ba, Katedra Ekologij, Narodowy Uniwersytet Transportu w Kijowi, Ukraina. ${ }^{3}$ Mgr Vadym Ziuziun, Katedra Ekologij, Narodowy Uniwersytet Transportu w Kijowi, Ukraina.
} 
Поэтому целью работы есть разработка подходов к формированию системы показателей эффективности программы экологической логистики транспортного предприятия.

Для достижения данной цели в работе необходимо рассмотреть общие подходы и методы к оценке качества выполнения программы; проанализировать особенности внедрения программы экологической логистики транспортных предприятий; разработать основные показатели оценки эффективности реализации программы.

\section{2. ОСОБЕННОСТИ ФОРМИРОВАНИЯ СИСТЕМЫ ПОКАЗАТЕЛЕЙ ЭФФЕКТИВНОСТИ ПРОГРАММЫ}

Под системой показателей эффективности программы будем понимать совокупность показателей, разработанных для нескольких уровней управления реализацией программы. При построении такой системы целесообразно поделить все показатели на несколько групп. В табл. 1 представлено три группы показателей эффективности программы.

Таблица 1. Классификация показателей эффективности программы

\begin{tabular}{|c|c|c|}
\hline Наименование группы & Пример показателя & Стратегия \\
\hline $\begin{array}{l}\text { Общие индикативные } \\
\text { показатели выполнения } \\
\text { программы }\end{array}$ & $\begin{array}{l}\text { Степень достижения цели } \\
\text { программы; степень } \\
\text { выполнения задач программы; } \\
\text { уровень снижения количества } \\
\text { отходов }\end{array}$ & $\begin{array}{l}\text { В общем виде } \\
\text { характеризуют } \\
\text { достижение } \\
\text { стратегических целей } \\
\text { выполнения программы } \\
\end{array}$ \\
\hline $\begin{array}{l}\text { Показатели } \\
\text { проекта, } \\
\text { ориентированные } \\
\text { выполнение } \\
\text { программы } \\
\text { надач } \\
\end{array}$ & $\begin{array}{l}\text { Степень приоритетности } \\
\text { проекта, степень значимости } \\
\text { направления по улучшению } \\
\text { состояния окружающей среды; } \\
\text { степень } \\
\text { эффективности проекта }\end{array}$ & $\begin{array}{l}\text { Характеризуют } \\
\text { критерии выбора } \\
\text { проектов для решения } \\
\text { задач достижения } \\
\text { стратегических целей } \\
\text { программы }\end{array}$ \\
\hline $\begin{array}{l}\text { Показатели выполнения } \\
\text { проекта, которые } \\
\text { характеризуют } \\
\text { операционную } \\
\text { эффективность } \\
\text { реализации программы }\end{array}$ & $\begin{array}{l}\text { Освоенный объем; время } \\
\text { реализации проекта, уровень } \\
\text { рисков в проекте; изменение } \\
\text { экологических аспектов; } \\
\text { эффективность коммуникаций } \\
\text { в проекте и т.д. }\end{array}$ & $\begin{array}{l}\text { Характеризуют } \\
\text { решение } \\
\text { достижения } \\
\text { стратегических целей }\end{array}$ \\
\hline
\end{tabular}

В первую группу условно отнесены общие индикативные показатели определения эффективности выполнения программы. В целом, они могут характеризовать достижение стратегических целей программы. Но с их помощью невозможно анализировать отдельные процессы и принимать конкретные управленческие решения.

Вторая группа представляет собой совокупность показателей, которые непосредственно характеризуют критерии выбора отдельных проектов для выполнения заданий достижения стратегических целей программы, т.е. ориентированных на реализацию стратегии. Важно отметить, что эти показатели отражают результат достижения отдельных подцелей программы на основе 
решения конкретной задачи. Если стратегия программы выражена нечетко, то и показатели этой группы будут, вероятно, индикативными.

Показатели третьей группы характеризуют операционную эффективность реализации отдельного проекта. Их можно рассчитать даже при отсутствии стратегии программы.

Такая классификация необходима для проверки и уточнения системы показателей, которая может быть использована для анализа программы. Следует отметить, что четких границ между указанными выше группами не существует. Один показатель может характеризовать как степень достижения стратегических целей, так и операционную эффективность.

Предположим, что уже получено (построено, синтезировано) набор показателей оценки эффективности программы:

$$
\Pi_{k}=\left\{\pi_{k_{1}}, \pi_{k_{2}}, \ldots \ldots, \pi_{k_{j k}}\right\}, \quad 1 \leq J_{k} \leq J_{K}, 1 \leq k \leq K,
$$

где $\Pi_{k}$ - интегрированный показатель эффективности программы;

$\pi_{k_{1}}, \pi_{k_{2}}, \ldots . ., \pi_{k_{J k}}-$ множество значений показателей на заданном уровне управления;

$K$ - количество уровней управления программой;

$k$ - текущий уровень управления, принимаем, что $1 \leq k \leq K<15 \div 20$;

$J-$ количество частичных показателей на заданном уровне управления.

Проведем анализ методов, которые позволят определить набор показателей эффективности программы экологической логистики транспортного предприятия.

\section{3. ОБЩИЕ ИНДИКАТИВНЫЕ ПОКАЗАТЕЛИ ВЫПОЛНЕНИЯ} ПРОГРАММЫ

Программно-целевой метод рассматривается шесть типов показателей выполнения программ. Из этих шести типов, четыре определены как основные и более весомые для оценки достигнутых результатов. Основные показатели выполнения программ - показатель затрат (входных ресурсов), показатель продукта, показатель производительности (эффективности), показатель результативности (качества). Вспомогательные показатели выполнения программ: показатель рабочей нагрузки, показатель полезности [2]. Набор показателей может быть представлен в виде следующей модели:

$$
\begin{gathered}
\Pi C=\left(\Pi_{1}, \Pi_{2}, \Pi_{3}, \Pi_{4}, \Pi_{5}, \Pi_{6}\right)=\left(\left\{\pi_{1}\right\} ;\left\{\pi_{2}\right\} ;\left\{\pi_{3}\right\} ;\left\{\pi_{4}\right\} ;\left\{\pi_{5}\right\} ;\left\{\pi_{6}\right\}\right)= \\
=\left(\pi_{1}, \pi_{2}, \pi_{3}, \pi_{4}, \pi_{5}, \pi_{6}\right) \\
0 \leq \pi_{i}^{-} \leq \pi_{i} \leq \pi_{i}^{+}<+\infty, i=1,2,3,4,5,6 .
\end{gathered}
$$

где ПС - показатель эффективности программы по программно-целевому методу; 
$\Pi_{1}$ - показатель затрат, который отображает структурированный объем входящих ресурсов, необходимый для осуществления соответствующего задания программы.

$\Pi_{2}$ - показатель продукта - это показатель, определяющий объем произведенной продукции или предоставленных услуг в ходе выполнения программы, количество пользователей товарами (работами, услугами) и т.д.

$\Pi_{3}$ - показатель производительности (эффективности). Являются мерой стоимости ресурсов на единицу продукта, и используются при определении и оценке различных методов предоставления услуг. Производительность - это отношение количественного показателя оказанной услуги к затратам в денежном или рабочем эквиваленте, необходимых для оказания услуги.

$\Pi_{4}$ - показатель результативности (качества). Определяет результаты проделанной работы и качество предоставляемых услуг. Позволяют оценить конечный эффект от осуществления программы, обеспечивает возможность отслеживать ежегодный прогресс в достижении цели программы и реализации ее задач.

$\Pi_{5}$ - показатель рабочей нагрузки. Определяет объем работы, который необходимо выполнить, или объем услуг, который следует предоставить.

$\Pi_{6}$ - показатель полезности. Индикатор, который показывает пользу от реализации данной программы для общества.

$\pi^{-}, \pi^{+}$- границы изменения показателя.

Согласно методологии управления программами Р2M [3], наиболее целесообразными и эффективными методами, формирования системы показателей эффективности программы являются метод “Пять “Е” и два "А”.

Метод "Пять "Е” и два "A" включает показатели: пять "E" - efficiency, effectiveness, earned value, ethics, ecology и два "A" - accountability, acceptability. Им все чаще используется внедрении программного управления. Набор показателей может быть представлен в виде следующей модели:

$$
\begin{aligned}
& \Pi 5 E 2 A=\left(E_{1}, E_{2}, E_{3}, E_{4}, E_{5}, A_{1}, A_{2}\right)= \\
& =\left(\left\{e_{1}\right\} ;\left\{e_{2}\right\} ;\left\{e_{3}\right\} ;\left\{e_{4}\right\} ;\left\{e_{5}\right\} ;\left\{a_{1}\right\} ;\left\{a_{2}\right\}\right)=, \\
& =\left(e_{1}, e_{2}, e_{3}, e_{4}, e_{5}, a_{1}, a_{2}\right) \\
& \quad 0 \leq e_{i}^{-} \leq e_{i} \leq e_{i}^{+}<+\infty, i=1,2,3,4,5 \\
& \quad 0 \leq a^{-} \leq a_{i} \leq a^{+}<+\infty, i=1,2 .
\end{aligned}
$$

где П5Е2A -показатель эффективности программы по методу «Пять «Е»и два «A»»;

$E_{l}$ - эффективность использования ресурсов в проектах - определяется отношением полученных от проекта выгод к количеству использованных ресурсов;

$E_{2}$ - результативность относится к удовлетворенности заинтересованных сторон до и после проекта;

$E_{3}$ - освоенный объем - универсальный критерий измерения прогресса проекта, в котором замысел проекта связан с его графиком (расписанием) и затратами 
(ресурсами);

$E_{4}$ - соблюдение этических норм - это реакция сообщества программы на общую приемлемость и социальную направленность идеи программы, на соблюдение в ее рамках социальных и корпоративных правил и оправдание этических ожиданий участников;

$E_{5}$ - экологичность - критерий поддержания непрерывного роста организации или непрерывного прогресса программы, при котором должное внимание обращается на защиту окружающей среды;

$A_{1}$ - надежность, определяется уровнем ответственности менеджмента за результаты проекта / программы, включая промежуточные результаты, получаемые заинтересованными сторонами, а также прозрачностью, наглядностью и открытостью (публичностью) при информировании ванной общественности о статусе проекта / программы на текущий момент;

$A_{2}$ - допустимость определяется целым рядом условий, которые приняли заинтересованные стороны о стоимостных показателях программы, выраженных в количестве вложенного капитала, гарантиях возврата инвестиций и утвержденным планам распределения потока денежных средств программы во времени;

$e^{-}, e^{+}, a^{-}, a^{+}$- границы изменения показателя.

Эффективным методом анализа достижения стратегических целей программы является использование сбалансированной системы показателей (BSC), которая была предложена профессором Harvard Business School Робертом Каплан (Dr. Robert S. Kaplan) и президентом консалтинговой фирмы Renaissance Solutions Дэвидом Нортоном (David P. Norton) [4]. Основной целью авторов было выявление новых способов повышения эффективности деятельности и достижения целей бизнеса. Одним из главных принципов, который обеспечил открытие новых возможностей - это целенаправленный учет не только финансовых показателей, но и других, которые в текущем измерении не считаются приоритетными.

$$
\begin{aligned}
& S P=\left(S P_{1}, S P_{2}, S P_{3}, S P_{4}\right)=\left(\left\{s p_{1}\right\} ;\left\{s p_{2}\right\} ;\left\{s p_{3}\right\} ;\left\{s p_{4}\right\}\right)= \\
& =\left(s p_{1}, s p_{2}, s p_{3}, s p_{4}\right) \\
& 0 \leq s p_{i}^{-} \leq s p_{i} \leq s p_{i}^{+}<+\infty, i=1,2,3,4 .
\end{aligned}
$$

где SP - показатель эффективности программы методом сбалансированной системы показателей;

$\mathrm{SP}_{1}$ - финансовая составляющая;

$\mathrm{SP}_{2}$ - клиентская составляющая;

$\mathrm{SP}_{3}$ - составляющая внутренних бизнес-процессов;

$\mathrm{SP}_{4}$ - составляющая обучения и развития персонала;

$s p^{-}, s p^{+}$- границы изменения показателя.

Оптимальным соотношением числа показателей для целей каждой из перспектив на стратегической карте является такое соотношение: финансы - 4-5 показателей $(22 \%)$; клиенты - 4-5 показателей $(22 \%)$; внутренние бизнес-процессы - 8-10 показателей (34\%); обучение и развитие - $4-5$ показателей (22\%). 
Обобщенная модель оценки эффективности программы может быть представлена множеством $\Pi C \cup S P$ или $П 5 E 2 A \cup S P$. Причем множеством $\Pi C \cup$ $S P$ может быль более эффективно использовано для оценки региональных муниципальных бюджетных программ. Множество П5E2A SP более универсально и может использоваться для оценки широкого круга различных программ.

Для формирования системы показателей эффективности программы экологической логистики транспортного предприятия рассмотрим особенности разработки и внедрения данной программы.

\section{4. ОСОБЕННОСТИ ПРОГРАММЫ ЭКОЛОГИЧЕСКОЙ ЛОГИСТИКИ АВТОТРАНСПОРТНОГО ПРЕДПРИЯТИЯ}

Жизненный цикл проекта внедрения системы экологической логистики транспортного предприятия включает стадии инициации, планирования, исполнения, контроля и закрытия.

Началом проекта внедрения системы экологической логистики является момент принятия руководством решения про экологизацию логистической системы предприятия.

На стадии инициации проводятся необходимые подготовительные работы.

Фаза планирования включает действия по проведению анализа производственных процессов, процессов закупки, распределения, складирования, транспортировки, обмена информацией и управления запасами на микро- и макрологистических уровнях. Важным элементом экологической логистики является формирование логистической системы управления отходами предприятия.

Внедрение проекта включает оптимизацию материальных, финансовых и информационных потоков; разработку документации системы; выбор транспортных средств с минимальными выбросами вредных веществ в атмосферу и наиболее экологически и социально безопасных маршрутов; внедрение управленческих мер улучшения организационной деятельности предприятия; внедрение производственно-технических мероприятий по снижению негативного влияния транспортного предприятия на окружающую среду, внедрение программы обучения работников предприятия.

Мониторинг и контроль проекта осуществляется постоянным контролем материальных, финансовых и информационных потоков.

Сроком окончания проекта может быть достижение определенных ранее экологических показателей производственной деятельности, определяющие изменения состояния окружающей среды.

Креативный шаблон проекта, учитывающий условия и ограничения реализации проекта внедрения экологически направленной ЛС, приведен в табл. 2.

Модель П5E2A $\cup S P$ учитывает все уровни показателей управления программой - общие индикативные показатели выполнения программы; показатели выбора проекта, ориентированные на выполнение задач программы и показатели выполнения проекта, которые характеризуют операционную эффективность реализации программы. 
Таблица 2. Креативный шаблон проекта внедрения системы экологической логистики предприятия

\begin{tabular}{|c|c|}
\hline $\begin{array}{l}\text { Проблема, на решение } \\
\text { которой } \\
\text { проект }\end{array}$ & $\begin{array}{l}\text { Высокий уровень негативного влияния на ОС, который } \\
\text { осуществляет предприятие. Увеличение расходов на преодоление } \\
\text { последствий загрязнения. Снижение конкурентоспособности } \\
\text { предприятия и международного имиджа }\end{array}$ \\
\hline Сфера деятельности & Управления деятельностью транспортного предприятия \\
\hline Предмет деятельности & $\begin{array}{l}\text { Управление материальными, финансовыми и информационными } \\
\text { потоками предприятия. Организационная структура. Финансовая } \\
\text { система. }\end{array}$ \\
\hline Миссия проекта & $\begin{array}{l}\text { Постоянное снижение загрязнения окружающей среды, } \\
\text { повышение экологической безопасности перевозок, реализация } \\
\text { энерго- и ресурсосберегающей производственно-хозяйственной } \\
\text { деятельности для достижения конкурентных преимуществ } \\
\text { предприятия на рынке транспортных услуг за счет внедрения } \\
\text { системы экологической логистики. }\end{array}$ \\
\hline Продукт проекта & $\begin{array}{l}\text { Разработанная и внедренная ЛС оптимального управления } \\
\text { материальными, финансовыми и информационными потоками, в } \\
\text { том числе в процессах обращения с отходами предприятия }\end{array}$ \\
\hline Целевая аудитория & $\begin{array}{l}\text { Работники предприятия, партнеры, клиенты, органы местного } \\
\text { самоуправления, население региона }\end{array}$ \\
\hline Цель проекта & $\begin{array}{l}\text { Внедрение экологически } \\
\text { уаправленной ЛС } \\
\text { управления материальными, финансовыми и информационнымо } \\
\text { потоками, в том числе в процессах обращения с отходами } \\
\text { предприятия }\end{array}$ \\
\hline Задачи проекта & $\begin{array}{l}\text { 1. Провести анализ существующей ЛС - производственных } \\
\text { процессов, процессов закупки, распределения, складирования, } \\
\text { транспортировки, обмена информацией и управления запасами на } \\
\text { микро- и макрологистических уровнях. } \\
\text { 2. Провести анализ системы обращения с отходами предприятия. } \\
\text { 3. Разработать цели, задачи и программу внедрения экологически } \\
\text { ориентированной ЛС. } \\
\text { 4. Разработать документацию управления материальными, } \\
\text { финансовыми и информационными потоками. } \\
\text { 5. Внедрить управленческие меры улучшения организационной } \\
\text { деятельности предприятия, в том числе управления отходами. } \\
\text { 6. Внедрить производственно-технические мероприятия по } \\
\text { снижению негативного воздействия предприятия на окружающую } \\
\text { среду. } \\
\text { 7. Внедрить программу обучения работников предприятия. } \\
\text { 8. Провести анализ сформированной ЛС. }\end{array}$ \\
\hline $\begin{array}{l}\text { Источники } \\
\text { финансирования }\end{array}$ & $\begin{array}{l}\text { Средства предприятия, банковские кредиты, возможно средства } \\
\text { Фонда охраны окружающей среды и средства муниципального } \\
\text { бюджета }\end{array}$ \\
\hline Срок внедрения & 3 года \\
\hline
\end{tabular}

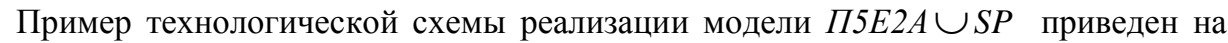
рис. 1. 


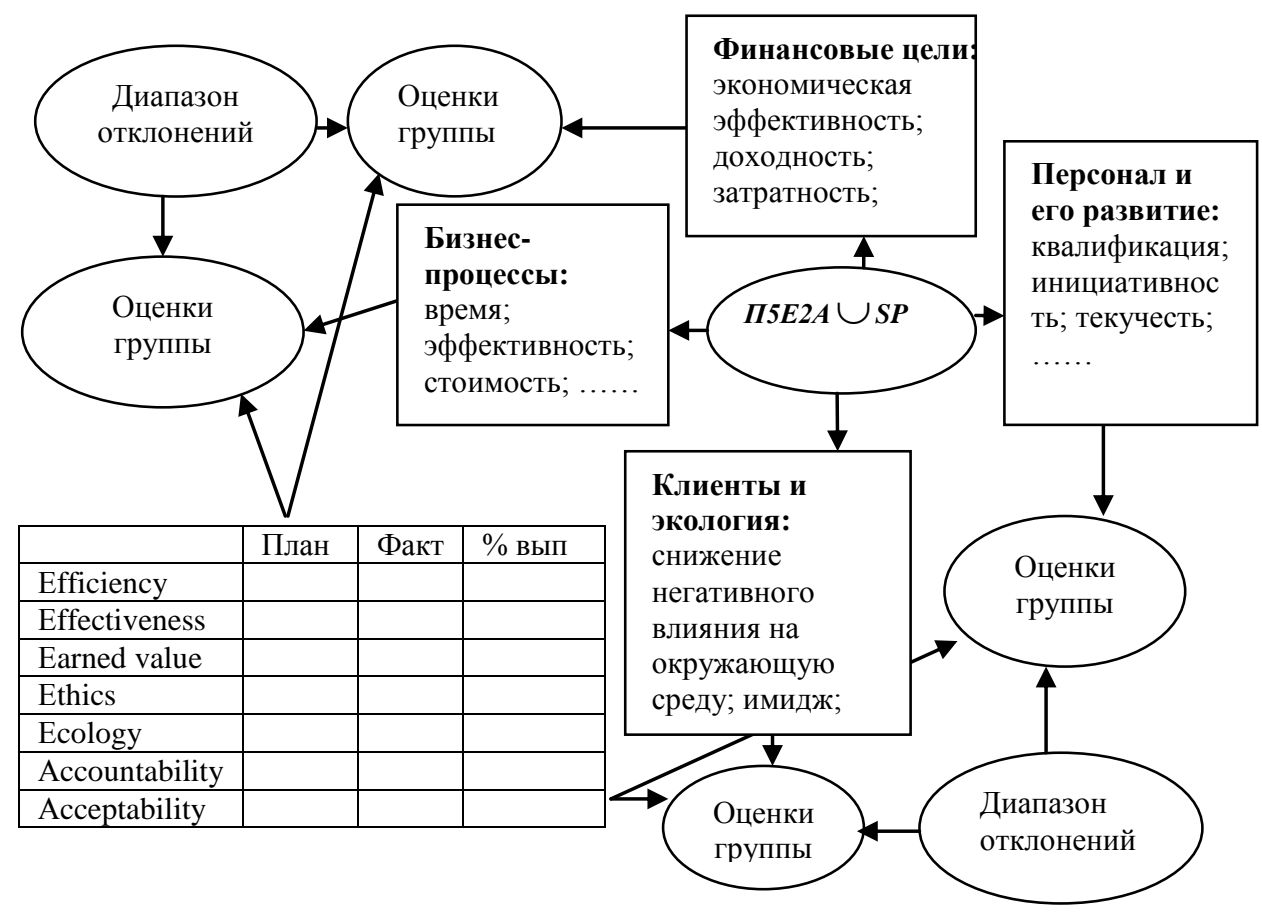

Рис.1. Пример технологической схемы реализации модели П5E2A $\cup P$

Этапы формирования системы показателей программы по модели П5E2A $S P$ включают:

1. Конкретизацию стратегических целей предприятия.

2. Связывание стратегических целей причинно-следственными цепочками.

3. Выбор показателей и определение их целевых значений.

4. Определение связи показателей с бизнес-процессами.

5. Определение стратегических мероприятий в виде конкретных проектов.

6. Сбор, оценка и анализ информации о выполнении стратегии.

Используем модель П5E2A $S P$ для формирование карты сбалансированных показателей эффективности программы экологической логистики транспортного предприятия.

\section{5. ФОРМИРОВАНИЕ КАРТЫ СБАЛАНСИРОВАННЫХ ПОКАЗАТЕЛЕЙ ЭФФЕКТИВНОСТИ ПРОГРАММЫ ЭКОЛОГИЧЕСКОЙ ЛОГИСТИКИ ТРАНСПОРТНОГО ПРЕДПРИЯТИЯ}

Основными целями стратегии внедрения программы экологической логистики могут стать:

1. Повышение прибыли предприятия за счет эффективной организации процессов перевозок, ремонта; снижение простоя и сокращения выплат и штрафов за загрязнение окружающей среды: 
- снижение суммарных операционных логистических затрат, в том числе в процессах обращения с отходами предприятия;

- увеличение производительности логистической инфраструктуры за счет внедрения рециклинга отходов.

2. Повышение удовлетворенности потребителей качеством логистических услуг за счет использования экологически безопасных транспортных средств:

- повышение качества логистического сервиса за счет повышения его экологической эффективности и социальной ответственности;

- $\quad$ внедрение использования транспортных средств классов Евро 4 и Евро 5, альтернативных видов горючего;

- $\quad$ поддержание минимальных затрат при качественном уровне сервиса;

- $\quad$ использование в логистической деятельности схем аутсорсинга.

3. Обеспечение постоянного повышения качества работы логистической инфраструктуры и процессов:

- $\quad$ азаработка необходимой документации обеспечение работы системы мониторинга и контроля, в том числе и процессов обращения с отходами;

- внедрение производственно-технических мероприятий по снижению количества отходов предприятия;

- внедрение производственно-технических мероприятий по снижению негативного воздействия предприятия на окружающую среду.

- $\quad$ увеличение производительности основных логистических процессов.

4. Повышение эффективности работы управленческого и производственного персонала:

- формирование ответственного отношения работников к вопросам сохранения состояния окружающей среды;

- $\quad$ создание благоприятного рабочего климата.

Для каждой стратегической цели программы разрабатываются комплекс показателей для каждого уровня управления программой. Производится их количественное оценивание или нормирование. Если количество показателей достаточно большое, то с помощью экспертных методов устанавливается степень их важности для результатов программы. Производится выбор локальных показателей. Определяются диапазоны отклонений для каждого показателя.

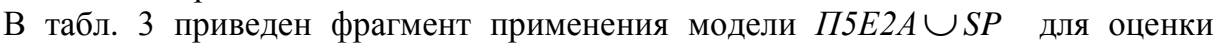
эффективности программы экологической логистики автотранспортного предприятия на стратегическом уровне управления программой. 
Таблица 3. Фрагмент применения BSC для оценки эффективности программы экологической логистики автотранспортного предприятия

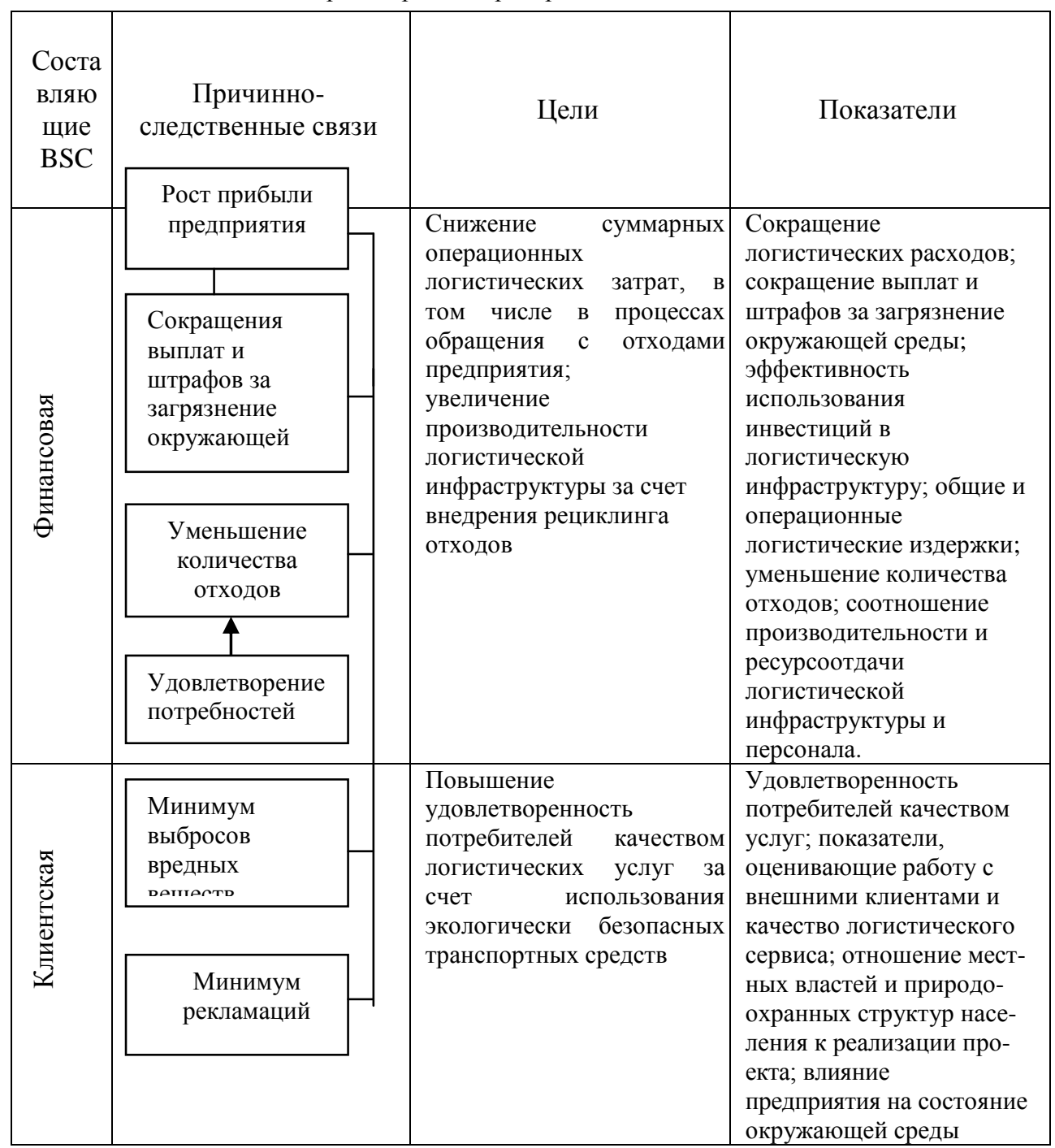




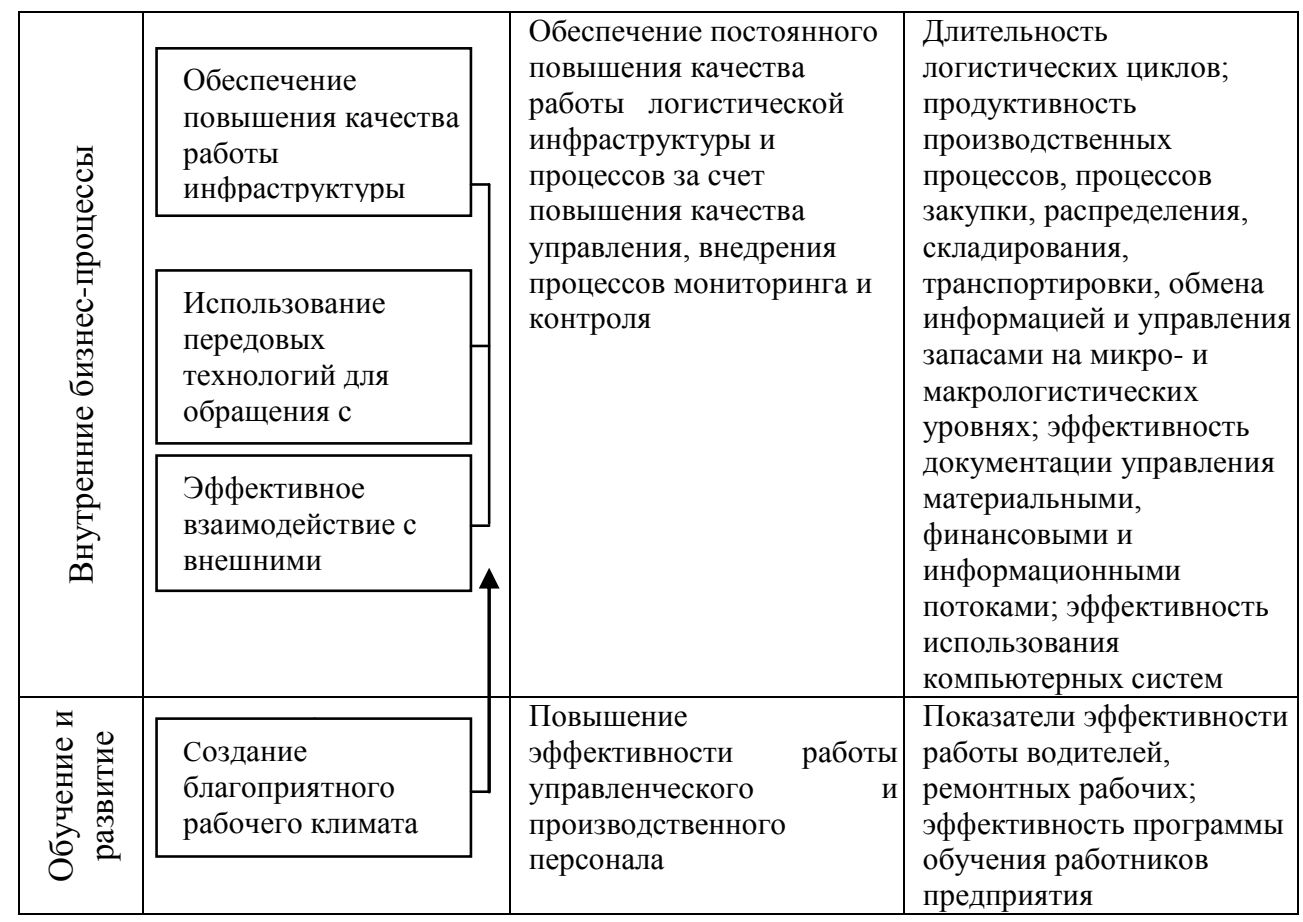

Приведем пример интеграции показателя $E_{l}$ - эффективность использования ресурсов в проектах с показателями финансовой составляющей программы сокращение логистических расходов; сокращение выплат и штрафов за загрязнение окружающей среды; эффективность использования инвестиций в логистическую инфраструктуру; общие и операционные логистические издержки; уменьшение количества отходов.

Показатель сокращения логистических расходов:

$$
E_{1} F_{1}=\frac{L_{1}}{L}
$$

где $L_{1}$ - сокращение логистических расходов,

$\mathrm{L}$ - затраты на внедрение экологической ЛC;

Показатель сокращение выплат и штрафов за загрязнение окружающей среды;

$$
E_{1} F_{2}=\frac{S H_{1}}{L},
$$

где $S H_{1}$ - сокращение выплат и штрафов за загрязнение окружающей среды.

Показатель эффективности использования инвестиций в логистическую инфраструктуру:

$$
E_{1} F_{3}=\frac{I n}{L},
$$

где In - эффективность использования инвестиций в логистическую инфраструктуру; 


$$
\begin{aligned}
& E_{1} F_{4}=\frac{I z_{1}}{L} ; \\
& E_{1} F_{5}=\frac{I z_{2}}{L}
\end{aligned}
$$

где $I z_{1}, I z_{2}$ - общие и операционные логистические издержки соответственно.

Аналогично определяются все остальные показатели, значение которых может изменяться и корректироваться в течение всего жизненного цикла программы.

Разработанная система показателей позволит эффективно управлять программой, ее стратегией, выбором наиболее эффективных проектов и контролировать непосредственную реализацию отдельных проектов и заданий на нижнем уровне реализации программы.

\section{6. ВЫВОДЫ}

Анализ общих подходов и методов оценки качества выполнения программы экологической логистики позволил выделить как наиболее эффективные показатели программно-целевого метода - показатель затрат (входных ресурсов), показатель продукта, показатель производительности (эффективности), показатель результативности (качества). Вспомогательные показатели выполнения программ: показатель рабочей нагрузки, показатель полезности.

Методология управления программами Р2М формирует систему показателей эффективности программы метод “Пять “Е” и два “А”, который включает показатели: пять "Е” - efficiency, effectiveness, earned value, ethics, ecology и два "А” - accountability, acceptability. Интеграция данных методологий с методикой сбалансированных показателей позволила на основании анализа особенностей внедрения программы экологической логистики транспортных предприятий, разработать основные показатели оценки эффективности реализации данной программы.

\section{ЛИТЕРАТУРА}

[1] Vasyl Mateichyk, Viktoria Hrut'ba, Natalia Gorid'ko. Program-target model of environmental logistics system of a transport enterprise. - Rachunkowść w logistyce przedsiębiorstw. - Rzeszów.- 2012. - P.84-94.

[2] Бєсєдін В. Ф. Прогнозування і розробка програм: метод. посіб. / В. Ф. Бєсєдін [та ін.] ; за ред В. Ф. Бєсєдіна. - К., 2000. - 468 с.

[3] Руководство по управлению инновацмонніми проектами и программами: т.1, версія 1.2 / пер. На рус.язык под. ред. С.Д.Бушуева.-К.: наук. світ, 2009.- 173 с.

[4] Каплан Роберт С., Нортон Дейвид П. Сбалансированная система показателей. От стратегии к действию. - М.: Олимп-Бизнес, 2003. - 304 с.

\section{ESTIMATION OF ECOLOGICAL LOGISTICS PROGRAM EFFICIENCY OF AN ENTERPRISE}

In the article the general approaches and methods of quality estimation of quality of programs implementation are considered. There were analyzed the features of introduction of the program on ecological logistics of transport enterprises. The basic indexes of estimation of efficiency of the program realization were worked out.

DOI: 10.7862/rz.2012.zim.24 